\title{
Performance of the FMF First-Trimester Preeclampsia-Screening Algorithm in a High-Risk Population in The Netherlands
}

\author{
Eva F. Zwertbroek ${ }^{a}$ Henk Groen ${ }^{b}$ Federica Fontanella ${ }^{a}$ Luana Maggio ${ }^{c}$ \\ Laura Marchid Caterina M. Bilardo $^{\mathrm{e}, \mathrm{f}}$ \\ a Obstetrics \& Gynaecology, University of Groningen, University Medical Center Groningen, Groningen, The \\ Netherlands; ${ }^{b}$ Epidemiology, University of Groningen, University Medical Center Groningen, Groningen, The

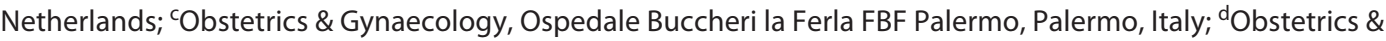

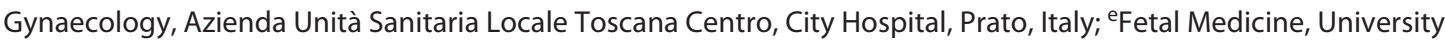 \\ Medical Centre, University of Groningen, Groningen, The Netherlands; ${ }^{f}$ Fetal Medicine, Amsterdam University \\ Medical Centers, Amsterdam, The Netherlands
}

\section{Keywords}

11-14 weeks scan · First-trimester screening · Preeclampsia · Biochemical markers · Uterine artery · Blood pressure

\begin{abstract}
Objective: The aim of the study was to evaluate the performance of the first-trimester Fetal Medicine Foundation (FMF) screening algorithm, including maternal characteristics and medical history, blood pressure, pregnancy-associated plasma protein $A$ and placenta growth factor, crown rump length, and uterine artery pulsatility index, for the prediction of preeclampsia in a high-risk population in the Netherlands. Methods: This is a prospective cohort including nulliparous women and women with preeclampsia or intrauterine growth restriction in previous pregnancy. We screened patients at 11-14 weeks of gestation to calculate the risk for preeclampsia. The primary outcome was preeclampsia and gestational age at delivery. Performance of the model was evaluated by area under the receiver operating characteristic (ROC) curves (AUCs) and calibration graphs; based on the ROC curves, optimal predicted risk cutoff values for our study population were defined. Results: We analyzed 362 women,
\end{abstract}

karger@karger.com www.karger.com/fdt

Karger $\frac{1}{\%}$

GOPEN ACCESS
(C) 2021 The Author(s)

Published by S. Karger AG, Basel

This is an Open Access article licensed under the Creative Commons Attribution-NonCommercial-4.0 International License (CC BY-NC) (http://www.karger.com/Services/OpenAccessLicense), applicable to the online version of the article only. Usage and distribution for commercial purposes requires written permission. of whom 22 (6\%) developed preeclampsia. The algorithm showed fair discriminative performance for preeclampsia $<34$ weeks (AUC $0.81 ; 95 \% \mathrm{Cl} 0.65-0.96$ ) and moderate discriminative performance for both preeclampsia $<37$ weeks (AUC $0.71 ; 95 \% \mathrm{Cl} 0.51-0.90$ ) and $<42$ weeks (AUC $0.71 ; 95 \%$ Cl 0.61-0.81). Optimal cutoffs based on our study population for preeclampsia $<34,<37$, and $<42$ weeks were 1:250, 1:64, and 1:22, respectively. Calibration was poor. Conclusions: Performance of the FMF preeclampsia algorithm was satisfactory to predict early and preterm preeclampsia and less satisfactory for term preeclampsia in a high-risk population. However, by addressing some of the limitations of the present study, the performance can potentially improve. This is essential before implementation is considered.

(c) 2021 The Author(s)
Published by S. Karger AG, Basel

\section{Introduction}

Preeclampsia is an important cause of maternal and perinatal morbidity and mortality, affecting about $2-10 \%$ of the pregnancies [1]. The condition is responsible for 
over 100,000 maternal deaths worldwide each year [2]. Preeclampsia is also associated with iatrogenic preterm birth, fetal growth restriction, and other neonatal morbidity, linked, in turn, to both short-term and long-term health consequences [3]. The only definitive treatment of preeclampsia is delivery of the baby. Therefore, screening and preventive treatment are important.

Recently, the ASPRE study showed that in women at high risk of preeclampsia, prophylactic low-dose aspirin started before 16 weeks of gestation reduces the rate of early preeclampsia with delivery $<32$ weeks by $90 \%$ and preterm preeclampsia with delivery $<37$ weeks by $60 \%$ [4]. They found no evidence that aspirin could reduce term preeclampsia. The use of aspirin also reduced the length of stay in the neonatal intensive care unit by $70 \%$, mainly due to reduction in very preterm delivery associated with early preeclampsia, prior to 32 weeks [5]. Administration of aspirin proved to be more effective when it is started in the first trimester [6]. Therefore, early detection of women at increased risk of preeclampsia is important to start prophylaxis as early as possible in order to reduce maternal and neonatal morbidity.

Currently, in The Netherlands, the risk profile for preeclampsia is determined at the first antenatal care visit, based on demographic characteristics and medical history. This is similar to guidelines in other countries such as the UK, where the National Institute for Health and Care Excellence (NICE) antenatal care guideline identifies women as being at high risk for preeclampsia when they have any one of the following risk factors: hypertensive disease in previous pregnancy, chronic hypertension, chronic renal disease, diabetes mellitus, or autoimmune disease. In addition, the presence of any 2 of the following moderate-risk factors also qualifies women as high-risk: nulliparity, age $\geq 40$ years, BMI $\geq 35 \mathrm{~kg} / \mathrm{m}^{2}$, family history of preeclampsia, or inter-pregnancy interval $>10$ years [7]. The American College of Obstetricians and Gynecologists (ACOG) considers women at high risk if they meet any of the following criteria: preeclampsia in previous pregnancy, chronic hypertension, chronic renal disease, diabetes mellitus, systemic lupus erythematosus or thrombophilia, nulliparity, age $>40$ years, $\mathrm{BMI} \geq 30 \mathrm{~kg} / \mathrm{m}^{2}$, family history of preeclampsia, or conception by in vitro fertilization [8]. The International Society for the Study of Hypertension in Pregnancy (ISSHP) recommends administration of aspirin prior to 16 weeks of gestation in women with "strong clinical risk factors," specified as prior preeclampsia, chronic hypertension, preexisting diabetes, maternal BMI $>30 \mathrm{~kg} / \mathrm{m}^{2}$, antiphospholipid syndrome, and assisted reproduction [9].
An alternative approach to predicting preeclampsia is by using the combination of maternal factors and biophysical tests and biomarkers to calculate a patient-specific risk [10-12]. Previous research has shown that preeclampsia with delivery at a certain gestational age can be predicted with good accuracy by a combination of maternal risk factors, mean arterial blood pressure, uterine artery Doppler, and maternal serum markers (pregnancyassociated plasma protein A [PAPP-A] and placenta growth factor [PlGF]) [13-16]. Screening for preeclampsia by the combination of the abovementioned factors at 11-13 weeks of gestation predicted $90 \%$ of early preeclampsia, $75 \%$ of preterm preeclampsia, and $42 \%$ of term preeclampsia at a screen-positive rate (SPR) of $10 \%[17$, 18]. These detection rates (DRs) were significantly better than those based on the NICE guideline, which had a DR for early preeclampsia of $46 \%$ at the SPR of $10 \%$. The ACOG guideline had a DR of $89 \%$ for preterm preeclampsia but with a SPR of $66 \%$ [19]. However, although the performance of the Fetal Medicine Foundation (FMF) algorithm seems superior to the mentioned guidelines, this algorithm to identify high-risk women eligible for treatment with aspirin needs to be validated in specific populations and settings before it can be implemented. Moreover, the ISSHP recommends investigating whether this first-trimester preeclampsia screening can be validated and integrated into the local antenatal health-care system [9]. Therefore, the aim of this pilot study was to evaluate the performance of the FMF algorithm for the prediction of preeclampsia in a high-risk Dutch obstetric care setting.

\section{Methods}

Study Design and Participants

This was a prospective cohort study to evaluate performance of the FMF preeclampsia-screening algorithm in the Netherlands [13-16]. Our study population consisted of pregnant women at a relatively high risk of preeclampsia. Both nulliparous women and women with preeclampsia or intrauterine growth restriction in a previous pregnancy were included in the study, regardless of the conception method. Twin pregnancies, maternal age below 18, and women on aspirin were excluded. This study was approved by the Institutional Review Board of the University Medical Centre Groningen (2013-216). Written informed consent was obtained at study entry.

\section{Study Procedures}

Pregnant women were asked by their primary health-care professional to participate in the study, at the time of the first visit or at counseling for prenatal screening. Potential trial participants were given both oral and written information. 
Women willing to participate were referred to the University Medical Centre Groningen for preeclampsia screening at 11-14 weeks of gestation. We recorded maternal characteristics (gestational age at the time of assessment, age, height, weight, ethnicity, and smoking status) and the general and obstetric medical history (parity, method of conception, presence of hypertensive disorders, diabetes mellitus, systemic lupus erythematosus, antiphospholipid syndrome, and presence of hypertensive disorders in the family). We measured the mean arterial pressure (twice per side) by automated devices and standardized protocol [20]. During the ultrasound scan, the fetal crown rump length was measured [21]. Transabdominal color Doppler ultrasonography was used to visualize both left and right uterine arteries, and the mean pulsatility index (PI) was calculated according to the FMF guideline [22]. To evaluate the learning curve of the uterine artery measurements, the duration of the examination was recorded and measurements were repeated (twice) by each operator and repeated by another sonographer, blind to the results of the previous [23]. A maternal blood sample was taken at the same visit. PAPP-A and PlGF were measured using PerkinElmer's AutoDelfia and expressed as multiple of median (MoM) [24]. The calculation of the risk of preeclampsia, taking all these demographic and biophysical variables into account, was performed by using the Astraia software package (version 1.20 .2 , copyright $2000-2010^{\circledR}$ Astraia Software GMBH).

Data regarding pregnancy outcomes were collected from hospital records and information from the midwives or general practitioners. For the hypertension cases, the gestational age at onset and type of hypertension (preeclampsia, gestational hypertension, or preexistent hypertension) were determined from obstetric records.

\section{Maternal and Neonatal Outcomes}

The primary end point was development of preeclampsia requiring delivery before 34 , before 37 , or before 42 weeks' gestation. Preeclampsia was defined according to the ISSHP [25]. The systolic blood pressure should be $140 \mathrm{~mm} \mathrm{Hg}$ or more and/or the diastolic blood pressure should be $90 \mathrm{~mm} \mathrm{Hg}$ or more on at least 2 occasions $4 \mathrm{~h}$ apart developing after 20 weeks of gestation in previously normotensive women. Moreover, there should be proteinuria of $300 \mathrm{mg}$ or more in $24 \mathrm{~h}$ or 2 readings of at least ++ on dipstick analysis of midstream or catheter urine specimens if no 24 -h collection is available.

Secondary end points were other adverse maternal outcomes of pregnancy (gestational hypertension, preexistent hypertension, hemolysis elevated liver enzymes and low platelets syndrome, placental abruption, and spontaneous delivery before 37 weeks of gestation). Neonatal secondary outcomes were stillbirth or neonatal death, intrauterine growth restriction (defined according to the Delfi consensus by Gordijn et al. [26]), intraventricular hemorrhage, necrotizing enterocolitis, respiratory distress syndrome, anemia resulting in blood transfusion, suspected or confirmed sepsis, and birth weight.

\section{Model Evaluation}

To assess the discriminative performance of the FMF model, receiver operating characteristic (ROC) curves were generated and areas under the curve (AUCs) were calculated. This was done for different gestational age windows for delivery with preeclampsia: before 34, before 37, or before 42 weeks. Calibration was assessed by plotting predicted probabilities against observed probabilities

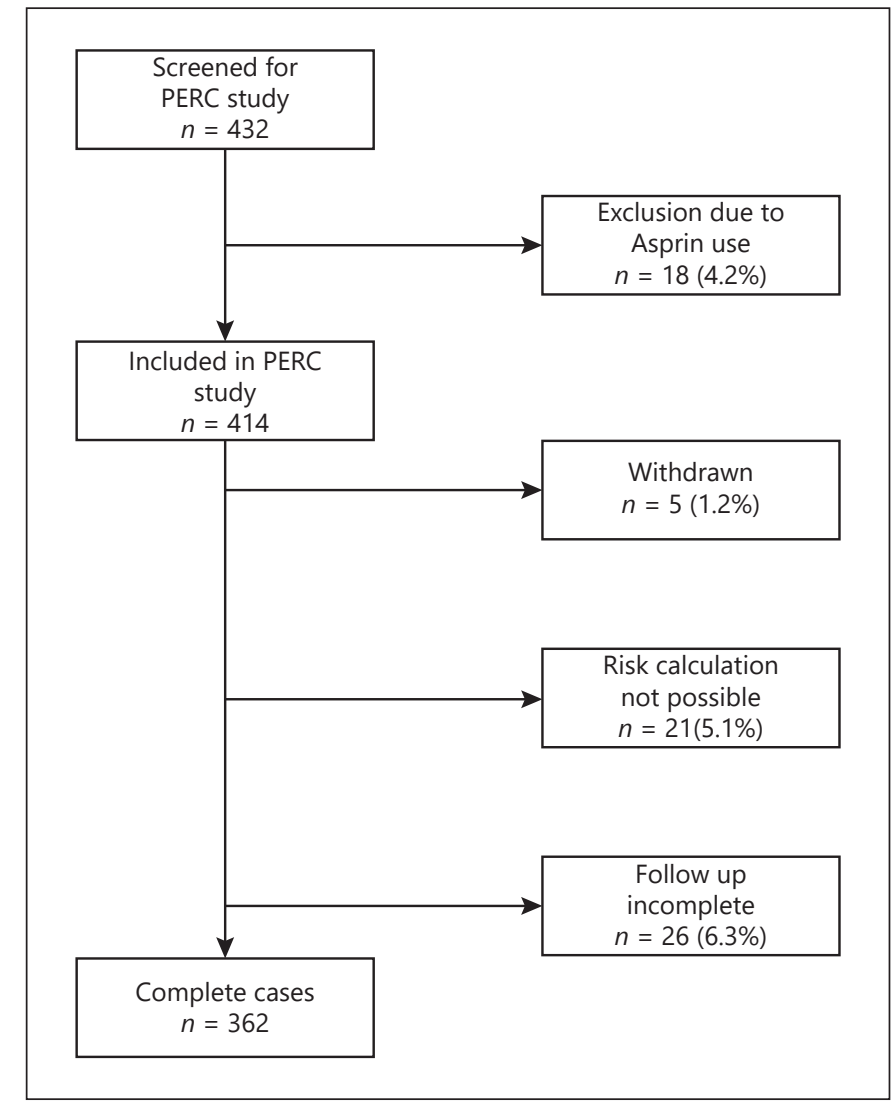

Fig. 1. Flowchart of inclusions.

in a calibration plot. STATA version 14 software was used for the model evaluation.

\section{Statistical Analysis}

Maternal characteristics, pregnancy details, and factors of the risk calculation algorithm were expressed in absolute numbers and percentages for dichotomous variables, and continuous variables were expressed in medians and interquartile ranges or mean and SDs as appropriate. The outcome measures were expressed as absolute numbers and percentages. To support the applied identification of high-risk women, clinical characteristics were compared between women with and without preeclampsia using $\chi^{2}$, Fishers exact test, $t$ test or Mann-Whitney U test as appropriate. The study was designed as a pilot study; therefore, no formal sample size calculation has been performed.

\section{Results}

A total of 432 women with singleton pregnancies were enrolled between June 2014 and December 2017. The screening was performed in the fetal medicine unit of the University Medical Center Groningen, the Neth- 
Table 1. Baseline characteristics

\begin{tabular}{|c|c|c|c|c|}
\hline Characteristic & $\begin{array}{l}\text { Total study population } \\
n=362\end{array}$ & $\begin{array}{l}\text { Preeclampsia } \\
n=22\end{array}$ & $\begin{array}{l}\text { Without preeclampsia } \\
n=340\end{array}$ & $p$ value \\
\hline \multicolumn{5}{|l|}{ Maternal characteristics } \\
\hline Age, years & $31(27-34)$ & $28(25-32)$ & $31(28-34)$ & 0.06 \\
\hline Caucasian, $n(\%)$ & $342(95.0)$ & $21(95.5)$ & $321(95.0)$ & 0.92 \\
\hline Smoking, $n(\%)$ & $22(6.1)$ & $1(4.5)$ & $21(6.2)$ & 0.76 \\
\hline Diabetes mellitus, $n(\%)$ & $7(1.9)$ & $0(0)$ & $7(2.1)$ & 0.79 \\
\hline Preexistent hypertension, $n(\%)$ & $14(3.9)$ & $2(9.1)$ & $12(3.6)$ & 0.19 \\
\hline Family history of PE, $n(\%)$ & $31(8.6)$ & $2(9.1)$ & $29(8.8)$ & 0.96 \\
\hline Systemic lupus erythematosus, $n(\%)$ & $2(0.6)$ & $0(0)$ & $2(0.6)$ & 0.71 \\
\hline Antiphospholipid syndrome, $n(\%)$ & $0(0)$ & $0(0)$ & $0(0)$ & na \\
\hline \multicolumn{5}{|l|}{ Pregnancy details, $n$ (\%) } \\
\hline \multicolumn{5}{|l|}{ Conception, $n(\%)$} \\
\hline Spontaneous & $284(78.5)$ & $21(95.5)$ & $263(77.4)$ & 0.87 \\
\hline Ovulation induction & $27(7.5)$ & $1(4.5)$ & $26(7.7)$ & \\
\hline ICSI & $24(6.7)$ & $0(0)$ & $24(7.1)$ & \\
\hline IVF & $14(3.9)$ & $0(0)$ & $14(4.1)$ & \\
\hline Gestational age, weeks & $13.0(12.4-13.4)$ & $12.9(12.3-13.5)$ & $13.0(12.4-13.4)$ & 0.65 \\
\hline Systolic blood pressure, $\mathrm{mm} \mathrm{Hg}$ & $116(109-122)$ & $120(112-133)$ & $115(109-123)$ & 0.04 \\
\hline Diastolic blood pressure, $\mathrm{mm} \mathrm{Hg}$ & $67(60-73)$ & $72(59-81)$ & $67(60-72)$ & 0.10 \\
\hline Fetal crown rump length, $\mathrm{mm}$ & $67.8(61.6-74.8)$ & $64(61-74)$ & $68(62-75)$ & 0.27 \\
\hline Uterine artery PI & $1.56(1.19-1.98)$ & $1.64(0.96-2.09)$ & $1.56(1.19-1.98)$ & 0.82 \\
\hline PAPP-A MoM & $0.91(0.58-1.32)$ & $0.83(0.47-1.22)$ & $0.93(0.58-1.34)$ & 0.36 \\
\hline PlGF MoM & $0.78(0.53-1.01)$ & $0.56(0.34-0.88)$ & $0.80(0.55-1.04)$ & 0.02 \\
\hline
\end{tabular}

$N$ (\%) or median (interquartile range). Ref: Tan et al. [19] Ultrasound 2018. IUGR, intrauterine growth restriction; ICSI, intracytoplasmatic sperm injection; IVF, in vitro fertilization; PAPP-A, pregnancy-associated plasma protein A; PlGF, placenta growth factor; MoM, multiple of median; PI, pulsatility index. ${ }^{\dagger}$ Risk cutoff $1: 150 .{ }^{\ddagger}$ Risk cutoff $1: 100$ is defined as high risk. ${ }^{\S}$ Cutoff $1: 10$.

erlands (Fig. 1). The women were either nulliparous or multiparous with a previous pregnancy complicated by fetal growth restriction or hypertensive disease. Of the screened women, 18 (4.2\%) cases had to be excluded because they were on low-dose aspirin. Five (1.2\%) women withdrew, and in 21 (5.1\%) cases, the risk calculation was not possible due to a missing variable. We were unable to collect pregnancy outcomes in $26(6.3 \%)$ cases, and these were considered as lost to follow-up. This resulted in 362 complete cases eligible for analysis of the performance of preeclampsia screening in the Dutch setting.

\section{Baseline Characteristics}

Baseline characteristics of our study population are shown in Table 1. Of the included women, $83.4 \%(n=$ $302)$ were nulliparous, $9.3 \%(n=40)$ had a previous pregnancy complicated by preeclampsia, and $7.7 \%(n=28)$ previously had a growth-restricted baby. Preexistent hypertension was present in $3.9 \%(n=14)$ of the cases. The screening was performed at a median gestational age of 13.0 (IQR 12.4-13.4) weeks.

\section{Maternal Outcomes}

Of the 362 women included in the study, $6.1 \%(n=22)$ developed preeclampsia. Women who developed pre- 

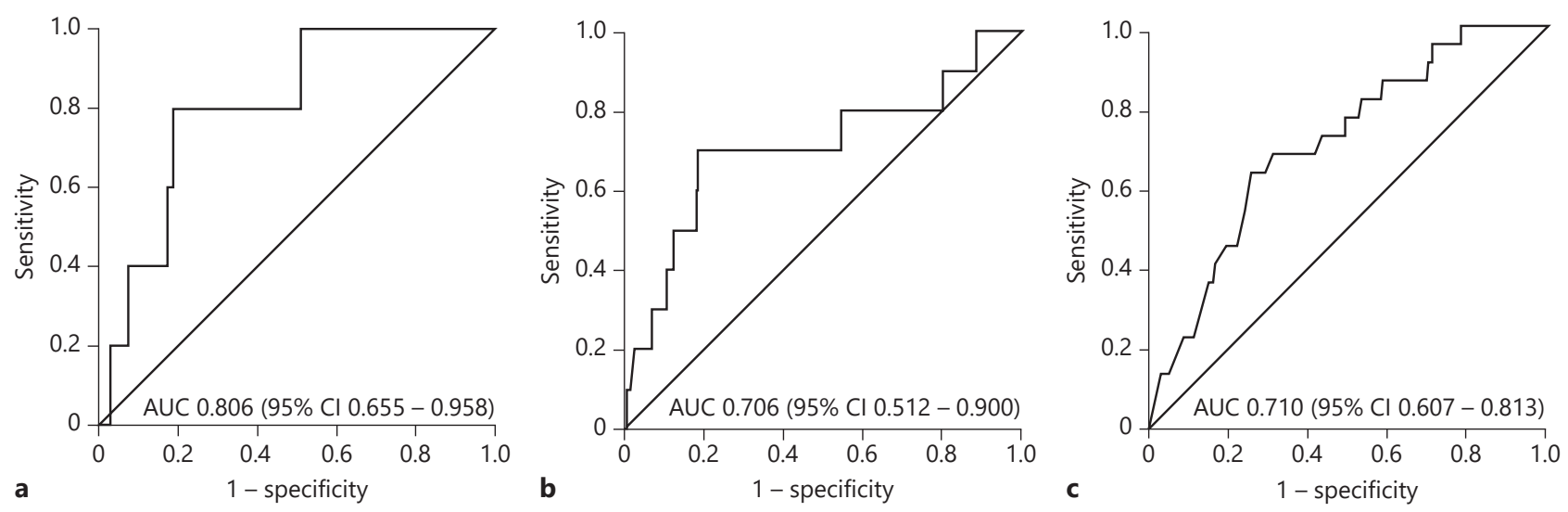

Fig. 2. ROC graph representing the discriminative value of the PE prediction algorithm. $\leq 34$ weeks (a), $\leq 37$ weeks (b), and $\leq 42$ weeks (c) of gestation. AUC, area under the curve; ROC, receiver operating characteristic.

eclampsia were slightly younger, more frequently nulliparous, had a higher systolic blood pressure at screening, and the PlGF MoM at 11-13 weeks was significantly lower (Table 1). There were no differences in uterine artery PI and PAPP-A MoM between women with and without preeclampsia. The median risk for developing preeclampsia at various gestational ages was, logically, increased for women who developed preeclampsia (Table 1).

Early-onset preeclampsia with delivery before 34 weeks of gestation occurred in $23 \%(n=5)$ of the women, and $46 \%(n=10)$ developed preeclampsia and delivered before 37 weeks. Other hypertensive pregnancy complications and neonatal outcomes are listed in Table 2.

\section{Performance of the Screening Algorithm for \\ Preeclampsia}

The FMF algorithm predicted preeclampsia requiring delivery before 34 weeks of gestation with good accuracy (AUC ROC 0.81, 95\% CI: 0.66-0.96). For preeclampsia with delivery up to 37 weeks of gestation, the model showed fair accuracy (AUC ROC 0.71), but with considerable uncertainty (95\% CI: 0.51-0.90). The performance of the algorithm to predict preeclampsia at any gestational age was similar to that of preterm preeclampsia (AUC ROC 0.71, 95\% CI: 0.61-0.81) (Fig. 2).

Based on the ROC curves, optimal high-risk cutoff values for prediction of preeclampsia before 34 weeks, before 37 weeks, or at any gestational age in this Dutch study population were $1: 250,1: 64$, and 1:22, respectively (Table 3 ). For these cutoffs, the algorithm showed a sensitiv-
Table 2. Maternal and neonatal outcomes

$\begin{array}{lc}\text { Outcome } & N(\%) \\ & n=362\end{array}$

Neonatal outcomes

Intrauterine growth restriction $27(7.4)$

RDS

$12(3.3)$

$9(2.6)$

NICU admission $24(6.6)$

Asphyxia $(\mathrm{pH}<7.05)$

Intraventricular hemorrhage $\quad 2(0.6)$

Necrotizing enterocolitis $0(0)$

Sepsis $18(5.0)$

HELLP, hemolysis elevated liver enzymes and low platelets; RDS, respiratory distress syndrome; NICU, neonatal intensive care unit. 
Table 3. Performance of preeclampsia screening in the Netherlands based on optimal risk cutoffs

\begin{tabular}{|c|c|c|c|c|c|c|c|c|c|c|c|c|}
\hline \multirow{2}{*}{$\begin{array}{l}\text { Delivery with } \\
\text { preeclampsia }\end{array}$} & \multicolumn{4}{|c|}{$<34$ weeks } & \multicolumn{4}{|c|}{$<37$ weeks } & \multicolumn{4}{|c|}{$<42$ weeks } \\
\hline & yes & & no & & yes & & no & & yes & & no & \\
\hline Screen - & 1 & $(20 \%)$ & 288 & $(81 \%)$ & 3 & $(30 \%)$ & 284 & $(81 \%)$ & 7 & $(32 \%)$ & 225 & $(68 \%)$ \\
\hline Total & 5 & $(1 \%)$ & 357 & $(99 \%)$ & 10 & $(3 \%)$ & 352 & $(97 \%)$ & 22 & $(6 \%)$ & 330 & (91\%) \\
\hline
\end{tabular}

High-risk cutoff for PE <34 weeks = 1:250; $\mathrm{PE}<37$ weeks = 1:64; $\mathrm{PE}<42$ weeks = 1:22 (optimal in our study population).

Table 4. Performance of preeclampsia screening in the Netherlands based on high-risk cutoffs as recommended by the FMF for administration of aspirin

\begin{tabular}{|c|c|c|c|c|c|c|c|c|}
\hline \multirow[t]{2}{*}{ Delivery with preeclampsia } & \multicolumn{4}{|c|}{$<34$ weeks } & \multicolumn{4}{|c|}{$<37$ weeks } \\
\hline & yes & & no & & yes & & no & \\
\hline Screen + & 2 & $(40 \%)$ & 56 & $(16 \%)$ & 7 & $(70 \%)$ & 84 & $(24 \%)$ \\
\hline Screen - & 3 & $(60 \%)$ & 301 & $(84 \%)$ & 3 & $(30 \%)$ & 268 & $(76 \%)$ \\
\hline Total & 5 & $(1 \%)$ & 357 & $(99 \%)$ & 10 & $(3 \%)$ & 352 & $(97 \%)$ \\
\hline
\end{tabular}

High-risk cutoff for PE $<34$ weeks $=1: 150 ; \mathrm{PE}<37$ weeks $=1: 100$ (as recommended by the FMF for administration of aspirin). FMF, Fetal Medicine Foundation.

ity and specificity of 80 and $81 \%$, respectively, for the prediction of early-onset preeclampsia. For preterm preeclampsia, sensitivity and specificity were 70 and $81 \%$, respectively. Both sensitivity and specificity decreased when predicting preeclampsia at any gestational age: 68 and $68 \%$ (Table 3 ). Positive and negative predictive values (PPV and NPV) for these optimal cutoffs were 5.6 and 99.7\% for prediction of preeclampsia $<34$ weeks, 9.3 and $99.0 \%$ for preeclampsia $<37$ weeks, and $12.5 \%$ and $97.0 \%$ for preeclampsia $<42$ weeks. The cutoffs of $1: 150$ for preeclampsia $<34$ weeks, and 1:100 for preeclampsia $<37$ weeks recommended by the FMF for administration of aspirin, showed a sensitivity of 40 and $70 \%$ and a specificity of 84 and $76 \%$, respectively, in our study population (Table 4). For this cutoff for preeclampsia <34 weeks, PPV and NPV were 3.4 and $99.0 \%$, respectively. The 1:100 cutoff for PE $<37$ weeks resulted in PPV and NPV of 7.7 and $98.9 \%$, respectively. DRs and false-positive rates (FPRs) for the optimal cutoff for this population and the FMF cutoffs used for administration of aspirin are displayed in Tables 3 and 4, respectively. In addition, we calculated DRs for fixed FPRs of 5, 10, and 25\%. DRs for the prediction of preeclampsia requiring delivery prior to 34 weeks were 20,40 , and $80 \%$ at FPR of 5,10 , and $25 \%$, respectively. The occurrence of preterm delivery with preeclampsia had DRs of 20,30 and $70 \%$ at a FPR of 5, 10, and $25 \%$, respectively. Term preeclampsia was predicted with DRs of 14,23 , and $83 \%$ at a FPR of 5, 10, and $25 \%$, respectively.

The graphic presentation of the calibration of the FMF preeclampsia screening algorithm is shown in Figure 3. The calibration of the algorithm in this cohort was poor. The model underestimated lower probabilities and overestimated higher probabilities.

\section{Discussion}

\section{Primary Findings and Conclusion}

In this pilot study, the performance of the most frequently used prediction algorithm for preeclampsia was satisfactory for early and preterm preeclampsia and less for late preeclampsia. However, the model showed poor calibration. We were able to define optimal cutoff values for prediction of preeclampsia before 34 weeks (1:250), before 37 weeks (1:64), and before 42 weeks (1:22) of gestation. With these cutoffs, we had a sensitivity of $80 \%$ and a specificity of $81 \%$ for early preeclampsia and 70 and $81 \%$ 


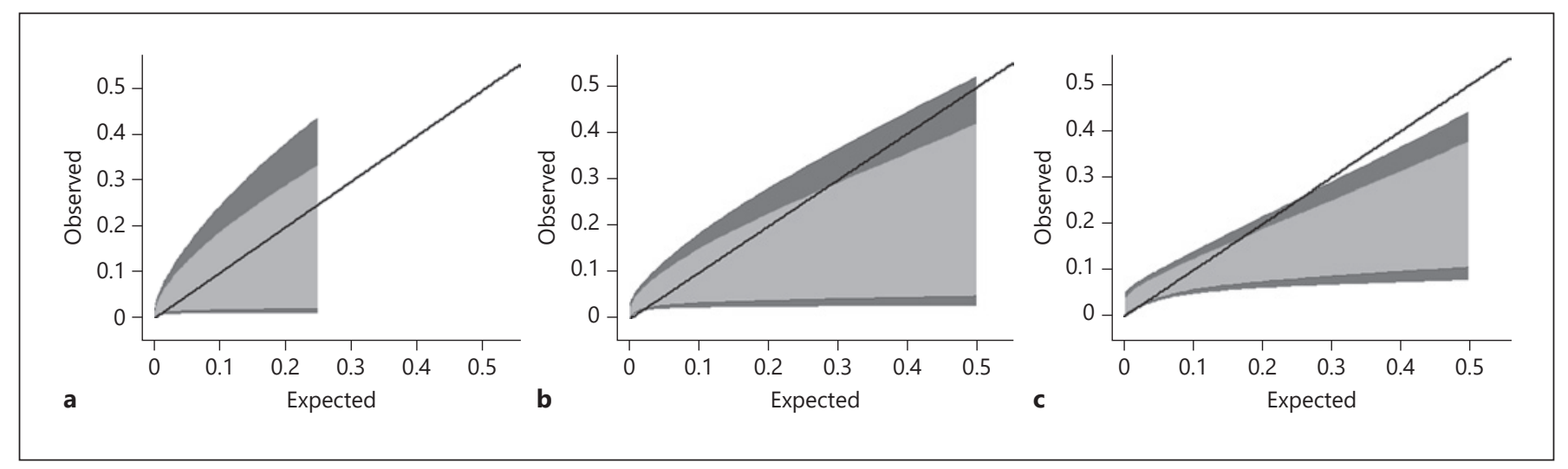

Fig. 3. Calibration graph for the prediction of preeclampsia. $\leq 34$ weeks (a), $\leq 37$ weeks (b), and $\leq 42$ weeks (c) of gestation. Legend: light gray area $=80 \%$ confidence interval; dark gray area $=95 \%$ confidence interval.

for preterm preeclampsia and a sensitivity of $68 \%$ and specificity of $68 \%$ for preeclampsia at any gestational age. Our results suggest that preeclampsia screening in the Netherlands is feasible, although the reasons for suboptimal performance of the screening should be addressed before successful implementation into clinical practice.

\section{Limitations}

This was a prospective cohort study, the first in The Netherlands addressing preeclampsia screening. The main limitation of the study is the small sample size. This leads to inevitable bias related to the lack of statistical power. In the Netherlands, preeclampsia screening is not yet part of routine clinical care. Currently, there is no routine visit or scan for nulliparous/healthy women at 11-13 weeks' gestation. Prior to 2017, the combined test was offered at 11-14 weeks' gestation as optional screening for aneuploidies. Uptake of this test in the Netherlands was notoriously low [27]. Currently, this test has been replaced by the cell-free fetal DNA and early scans, besides the dating scan at 10 weeks, are barely performed. In addition, low-risk pregnancies and all primigravidae are cared for by midwives. We found it arduous to motivate midwives in our region to enroll women in the study, as they were reluctant to offer to women a study taking place in a hospital (fear of medicalization). Additionally, many women with preeclampsia or a growth-restricted fetus in a previous pregnancy were excluded from the study because they were already using prophylactically low-dose aspirin, as they are mainly cared for by gynecologists.

The sonographers who measured the uterine artery in this study had obtained the FMF certificate for this measurement. To assess reliability of this important variable in the screening algorithm, we also performed a reproducibility study in the first 100 participants. The results of the reproducibility and repeatability of the uterine artery measurement were published previously and indicated that the inter- and intra-observer reproducibility was moderate [23]. Additionally, in the current study, uterine artery PI was not significantly associated with preeclampsia. Both findings indicate that the technique of uterine artery measurement was suboptimal, and this may have played a role in the underperformance of the preeclampsia screening in our study population. When the measurement is not performed routinely, it may be difficult to acquire the necessary experience.

\section{Comparison with Other Studies in the Literature}

The risk factors taken into account in the FMF preeclampsia-screening algorithm have been studied extensively [7]. The algorithm we used in this study has been tested and validated in multiple centers and countries $[16$, 18]. Results have shown a DR of $90 \%$ for early preeclampsia, $75 \%$ for preterm preeclampsia, and $41 \%$ for term preeclampsia, at a FPR of $10 \%$ [17]. AUCs were 0.96, 0.92, and 0.78 , respectively. In our validation study, we found a DR for early and preterm preeclampsia of 80 and $70 \%$, respectively, but a much higher FPR of $19 \%$ for both. The DR for preeclampsia at any gestational age was $68 \%$, at the cost of a FPR of $32 \%$. This performance is clearly inferior to that of other studies $[16,18]$. Cutoffs are known to be influenced by the risk characteristics of the study population. In our population, the optimal cutoff for preeclampsia before 37 weeks was 1:64, which is in accordance with the results of Tan et al. [19] (1:67), suggesting a similarity between our study population and theirs. 
In our study, the MoMs of both uterine artery PI and PAPP-A were not significantly different between women who did and did not develop preeclampsia. Other studies showed that the addition of uterine artery Doppler improved preeclampsia screening based on maternal characteristics from 45 to $63 \%$ [28]. Another study indicates that also uterine artery contributes significantly to the AUC and DR of this screening algorithm [16]. Both uterine artery PI MoM and PAPP-A MoM showed a linear relation with gestational age at delivery in preeclamptic women. Moreover, PAPP-A MoM, separately or in combination with the mean arterial blood pressure, was suggested as a predictor of preeclampsia [16]. However, recent studies have indicated that the current preeclampsiascreening algorithm, based on maternal, biophysical, and biochemical factors, does not significantly improve its performance after the addition of PAPP-A. The fact that our findings regarding PAPP-A and uterine artery Doppler are at variance with other studies may partly explain the suboptimal performance of the algorithm.

\section{Clinical Implications}

In spite of the suboptimal performance of the preeclampsia screening algorithm in our study, our results indicate that preeclampsia screening is feasible and could be implemented into clinical practice in the future. In addition, screening by calculating individual risks by maternal, biophysical, and biochemical markers seems superior to the current guidelines' approach by solitary maternal characteristics [19]. The definition of optimal risk cutoffs is important to identify women that should be offered low-dose aspirin prophylaxis. We identified as optimal cutoff for preterm preeclampsia 1:64, which is in keeping with the most recent validation study, which defined risk cutoffs for several sub populations [17]. Currently, a cutoff of 1:100 for preterm preeclampsia is used as criterion to recommend aspirin prophylaxis, in accordance with the findings of the ASPRE trial [4]. In a predominantly white population a cutoff of 1:150 is recommended [17]. These recommendations might increase DR, but could also lead to an increase in FPRs (Table 4). As a consequence, a large number of women would unnecessarily start with aspirin. It is reassuring that recent research has shown that an aspirin dose of $>100 \mathrm{mg}$ administered before 16 weeks of gestation has no negative effects in pregnancy and it decreases the risk of placental abruption or antepartum hemorrhage [6]. Hence, both risk cutoffs (1:64 and 1:100) could be adopted in our population. Recently, the minister of health has declared the intention to start a national implementation study on the value of a routine 13 weeks scan offered to all pregnant women. This scan would represent a unique opportunity for implementation of the preeclampsia screening algorithm. Aspirin $(150 \mathrm{mg} /$ day) could then be recommended to high-risk women from 13 weeks onwards [29].

\section{Future Research}

A large number of models assessing the role of different variables in preeclampsia screening are available. Further research should focus on defining the best algorithm that could to be implemented in different care systems and defining optimal cutoffs for aspirin administration [30]. A potential improvement in the DR could be obtained by automation of the uterine artery measurement, reducing inter- and intra-operator variation or, alternatively, the definition of an algorithm where measurement of the uterine artery PI is redundant. Future research could focus on cost-effectiveness of preeclampsia screening and implementation in different care systems leading to improved patient care.

\section{Conclusion}

In this pilot study in The Netherlands, performance of the FMF preeclampsia-screening algorithm was satisfactory to predict early and preterm preeclampsia and less satisfactory for term preeclampsia, albeit in a high-risk population. DRs and FPRs were fair, despite a low number of events. Further training and standardization of the technique of uterine artery Doppler measurement has the potential to improve screening performance. This prospective pilot study showed that performance and implementation of preeclampsia screening in the decentralized Dutch care system could be feasible.

\section{Acknowledgements}

The authors thank the patients, sonographers, research nurses, and lab workers.

\section{Statement of Ethics}

Our research complies with the guidelines for human studies and was conducted ethically in accordance with the World Medical Association Declaration of Helsinki. This study was approved by the Institutional Review Board of the University Medical Centre Groningen (2013-216). Written informed consent was obtained at study entry. 


\section{Conflict of Interest Statement}

The authors have no conflict of interest to declare.
H.G. All authors contributed to the interpretation of the data. All authors revised the manuscript and added their intellectual content. All authors approved the final submitted version.

\section{Author Contributions}

C.M.B. obtained funding for the study. H.G. and C.M.B. designed the study. E.F.Z. was responsible for data collection together with F.F., L.M. and L.M. E.F.Z. did the analysis together with

\section{Funding Sources}

The pilot study was funded by Doelmatigheidsfonds UMCG - healthy aging pilots (Bilardo 2010-674506).

\section{References}

1 Khan KS, Wojdyla D, Say L, Gülmezoglu AM, Van Look PF. WHO analysis of causes of maternal death: a systematic review. Lancet. 2006 Apr 1;367(9516):1066-74.

2 Hutcheon JA, Lisonkova S, Joseph KS. Epidemiology of pre-eclampsia and the other hypertensive disorders of pregnancy. Best Pract Res Clin Obstet Gynaecol. 2011 Aug;25(4):391-403.

3 Abalos E, Cuesta C, Carroli G, Qureshi Z, Widmer M, Vogel JP, et al. Pre-eclampsia, eclampsia and adverse maternal and perinatal outcomes: a secondary analysis of the World Health Organization Multicountry Survey on Maternal and Newborn Health. BJOG. 2014 Mar;121(Suppl 1):14-24.

4 Rolnik DL, Wright D, Poon LC, O'Gorman N, Syngelaki A, de Paco Matallana C, et al. Aspirin versus placebo in pregnancies at high risk for preterm preeclampsia. N Engl J Med. 2017 Aug 17;377(7):613-22.

5 Wright D, Rolnik DL, Syngelaki A, de Paco Matallana C, Machuca M, de Alvarado M, et al. Aspirin for evidence-based preeclampsia prevention trial: effect of aspirin on length of stay in the neonatal intensive care unit. Am J Obstet Gynecol. 2018 Jun;218(6):612-e6.

6 Roberge S, Villa P, Nicolaides K, Giguère Y, Vainio M, Bakthi A, et al. Early administration of low-dose aspirin for the prevention of preterm and term preeclampsia: a systematic review and meta-analysis. Fetal Diagn Ther. 2012;31(3):141-6.

7 National Collaborating Centre for Women's and Children's Health (UK). Hypertension in pregnancy: the management of hypertensive disorders during pregnancy. 2010 Aug.

8 Committee opinion summary No. 638: first-trimester risk assessment for early-onset preeclampsia. Obstet Gynecol. 2015 Sep;126(3):689.

9 Brown MA, Magee LA, Kenny LC, Karumanchi SA, McCarthy FP, Saito S, et al. Hypertensive disorders of pregnancy: ISSHP classification, diagnosis, and management recommendations for international practice. Hypertension. 2018 Jul;72(1):24-43.

10 Wright D, Akolekar R, Syngelaki A, Poon LC, Nicolaides KH. A competing risks model in early screening for preeclampsia. Fetal Diagn Ther. 2012;32(3):171-8.

11 Akolekar R, Syngelaki A, Poon L, Wright D, Nicolaides KH. Competing risks model in early screening for preeclampsia by biophysi$\mathrm{cal}$ and biochemical markers. Fetal Diagn Ther. 2013;33(1):8-15.

12 Wright D, Syngelaki A, Akolekar R, Poon LC, Nicolaides $\mathrm{KH}$. Competing risks model in screening for preeclampsia by maternal characteristics and medical history. Am J Obstet Gynecol. 2015 Jul;213(1):62-10.

13 Poon LC, Akolekar R, Lachmann R, Beta J, Nicolaides KH. Hypertensive disorders in pregnancy: screening by biophysical and biochemical markers at 11-13 weeks. Ultrasound Obstet Gynecol. 2010 Jun;35(6):662-70.

14 Akolekar R, Syngelaki A, Poon L, Wright D, Nicolaides KH. Competing risks model in early screening for preeclampsia by biophysi$\mathrm{cal}$ and biochemical markers. Fetal Diagn Ther. 2013;33(1):8-15.

15 Akolekar R, Syngelaki A, Sarquis R, Zvanca M, Nicolaides KH. Prediction of early, intermediate and late pre-eclampsia from maternal factors, biophysical and biochemical markers at 11-13 weeks. Prenat Diagn. 2011 Jan;31(1):66-74.

16 O'Gorman N, Wright D, Syngelaki A, Akolekar R, Wright A, Poon LC, et al. Competing risks model in screening for preeclampsia by maternal factors and biomarkers at 11-13 weeks gestation. Am J Obstet Gynecol. 2016 Jan;214(1):103-e12.

17 Tan MY, Syngelaki A, Poon LC, Rolnik DL, O'Gorman N, Delgado JL, et al. Screening for pre-eclampsia by maternal factors and biomarkers at 11-13 weeks' gestation. Ultrasound Obstet Gynecol. 2018 Aug;52(2):186-95.

18 O'Gorman N, Wright D, Poon LC, Rolnik DL, Syngelaki A, Wright A, et al. Accuracy of competing-risks model in screening for preeclampsia by maternal factors and biomarkers at 11-13 weeks' gestation. Ultrasound Obstet Gynecol. 2017 Jun;49(6):751-5.

19 Tan MY, Wright D, Syngelaki A, Akolekar R, Cicero S, Janga D, et al. Comparison of diagnostic accuracy of early screening for pre-eclampsia by NICE guidelines and a method combining maternal factors and biomarkers: results of SPREE. Ultrasound Obstet Gynecol. 2018 Jun;51(6):743-50.

20 Poon LC, Zymeri NA, Zamprakou A, Syngelaki A, Nicolaides KH. Protocol for measurement of mean arterial pressure at 11-13 weeks' gestation. Fetal Diagn Ther. 2012; 31(1):42-8.

21 Robinson HP, Fleming JE. A critical evaluation of sonar "crown-rump length" measurements. Br J Obstet Gynaecol. 1975 Sep;82(9):702-10.

22 Plasencia W, Maiz N, Bonino S, Kaihura C, Nicolaides KH. Uterine artery Doppler at 11 +0 to $13+6$ weeks in the prediction of preeclampsia. Ultrasound Obstet Gynecol. 2007 Oct;30(5):742-9.

23 Marchi L, Zwertbroek E, Snelder J, Kloosterman M, Bilardo CM. Intra- and inter-observer reproducibility and generalizability of first trimester uterine artery pulsatility index by transabdominal and transvaginal ultrasound. Prenat Diagn. 2016 Dec;36(13):1261-9.

24 Pandya P, Wright D, Syngelaki A, Akolekar R, Nicolaides KH. Maternal serum placental growth factor in prospective screening for aneuploidies at 8-13 weeks' gestation. Fetal Diagn Ther. 2012;31(2):87-93.

25 Brown MA, Lindheimer MD, de Swiet M, Van Assche A, Moutquin JM. The classification and diagnosis of the hypertensive disorders of pregnancy: statement from the International Society for the Study of Hypertension in Pregnancy (ISSHP). Hypertens Pregnancy. 2001; 20(1):IX-XIV.

26 Gordijn SJ, Beune IM, Thilaganathan B, Papageorghiou A, Baschat AA, Baker PN, et al. Consensus definition of fetal growth restriction: a Delphi procedure. Ultrasound Obstet Gynecol. 2016 Sep;48(3):333-9.

27 Bakker M, Birnie E, Pajkrt E, Bilardo CM, Snijders RJ. Low uptake of the combined test in The Netherlands: which factors contribute? Prenat Diagn. 2012 Dec;32(13):1305-12.

28 Papageorghiou AT, Yu CK, Erasmus IE, Cuckle HS, Nicolaides KH. Assessment of risk for the development of pre-eclampsia by maternal characteristics and uterine artery Doppler. BJOG. 2005 Jun;112(6):703-9.

29 Bujold E, Roberge S, Nicolaides KH. Lowdose aspirin for prevention of adverse outcomes related to abnormal placentation. Prenat Diagn. 2014 Jul;34(7):642-8.

30 Allen RE, Zamora J, Arroyo-Manzano D, Velauthar L, Allotey J, Thangaratinam S, et al. External validation of preexisting first trimester preeclampsia prediction models. Eur J Obstet Gynecol Reprod Biol. 2017 Oct;217:119-25. 